\title{
Mesenchymal Stem Cell Transplantation Promotes Functional Recovery through MMP2/STAT3 Related Astrogliosis after Spinal Cord Injury
}

\author{
Choonghyo Kim ${ }^{1}$, Hee Jung Kim${ }^{1}$, Hyun Lee ${ }^{2}$, Hanbyeol Lee ${ }^{3}$, Seung Jin Lee ${ }^{1}$, \\ Seung Tae Lee ${ }^{2}$, Se-Ran Yang ${ }^{3}$, Chun Kee Chung ${ }^{4}$ \\ ${ }^{I}$ Department of Neurosurgery, Kangwon National University School of Medicine, Chuncheon, Korea \\ ${ }^{2}$ Divisions of Applied Animal Science and Animal Resource Science, Department of Animal Life Science, Kangwon National University, \\ Chuncheon, Korea \\ ${ }^{3}$ Department of Thoracic and Cardiovascular Surgery, Kangwon National University School of Medicine, Chuncheon, Korea \\ ${ }^{4}$ Department of Neurosurgery, Seoul National University College of Medicine, Seoul, Korea
}

Background and Objectives: Treatment with mesenchymal stem cells (MSC) in spinal cord injury (SCI) has been highlighted as therapeutic candidate for SCI. Although astrogliosis is a major phenomenon after SCI, the role of astrogliosis is still controversial. In this study, we determined whether acute transplantation of MSC improves the outcome of SCI through modulating astrogliosis.

Methods: Bone marrow derived rat MSCs were induced neural differentiation and transplanted after acute SCI rats. Matrix metalloproteinase (MMP) and neuro-inflammatory pathway were analyzed for acute astrogliosis at 1, 3 and $7 \mathrm{~d}$ after SCI in RT-PCR- and western blot analysis. Functional outcome was assessed serially at postoperative $1 \mathrm{~d}$ and weekly for 4 weeks. Histopathologic analysis was undertaken at 7 and $28 \mathrm{~d}$ following injury in immunohistochemistry.

Results: Transplantation of MSCs decreased IL-1 $\alpha$, CXCL-2, CXCL-10, TNF- $\alpha$ and TGF- $\beta$ in a rat model of contusive SCI. Protein level of NF- $\kappa \mathrm{B}$ p65 was slightly decreased while level of STAT-3 was increased. In immunohistochemistry, MSC transplantation increased acute astrogliosis whereas attenuated scar formation with increased sparing white matter of spinal cord lesions. In RT-PCR analysis, mRNA levels of MMP2 was significantly increased in MSC transplanted rats. In BBB locomotor scale, the rats of MSC treated group exhibited improvement of functional recovery.

Conclusions: Transplantation of MSC reduces the inflammatory reaction and modulates astrogliosis via MMP2/STAT3 pathway leading to improve functional recovery after SCI in rats.

Keywords: Spinal cord injury, MSC transplantation, Astrogliosis, MMP2, STAT3

Received: December 15, 2018, Revised: May 14, 2019, Accepted: May 17, 2019, Published online: June 30, 2019

Correspondence to Chun Kee Chung

Department of Neurosurgery, Seoul National University Hospital, 101 Daehak-ro, Jongno-gu, Seoul 03080, Korea

Tel: +82-2-2072-2352, Fax: +82-2-744-8459, E-mail: chungc@snu.ac.kr

Co-Correspondence to Se-Ran Yang

Department of Thoracic and Cardiovascular Surgery, School of Medicine, Kangwon National University, 1 Kangwondaehak-gil, Chuncheon 24341, Korea

Tel: +82-33-250-7883, Fax: +82-33-255-8809, E-mail: seran.yang@gmail.com, seran@kangwon.ac.kr

(a) This is an open-access article distributed under the terms of the Creative Commons Attribution Non-Commercial License (http://creativecommons.org/licenses/by-nc/4.0/), which permits unrestricted non-commercial use, distribution, and reproduction in any medium, provided the original work is properly cited.

Copyright (c) 2019 by the Korean Society for Stem Cell Research 


\section{Introduction}

The global incidence of spinal cord injury (SCI) is estimated from 23 per million inhabitants $(1,2)$. Patients with SCI suffer from permanent impairment, limitation of activity, low quality of life, loss of job and increased medical cost (3). To overcome the permanent damage of SCI, many therapeutic approaches have been applied. Glial scar is known as the barrier to regenerated injured spinal cord, and it is formed from astrogliosis which is increased complex molecular and cellular responses, mainly morphological change and proliferation of astrocytes (4). In astrogliosis, various pro-inflammatory cytokines and matrix metalloproteinases (MMPs) were involved. In the brain and spinal cord, MMPs are involved to both injury and repair cellular mechanisms (5). In the spinal cord, MMP2 and MMP9 plays a pivotal role respectively to modulate astrocytes in the acute and chronic phases following SCI (6). Astrocytes in the spinal cord are most abundant cells and mainly contribute in glial scar formation. Reactive astrogliosis is initiated with stimulated astrocytes migration at the site of injury and characterized by increased expression of glial fibrillary acidic protein (GFAP). Mesenchymal stem cells (MSCs) or precursor cell had been highlighted as one of them from inhibition of astrogliosis. However, role of MSC on acute astrogliosis is not defined clearly because treatment with MSCs has been delayed due to avoiding an acute hostile environment $(7,8)$. In addition, several reports support the protective role of acute astrogliosis in SCI (9-12). Anderson et al. (9) reported astrocyte and acute gliosis improve regeneration of injured cord through decreasing scar formation and introducing axonal growth.

Inhibition of astrogliosis had been believed the key mechanism to recover from SCI by survived transplanted cells. These studies suggest that MSC treatment on acute astrogliosis improves during initial stage of recovery, and those evidences supprot to overcome adversities of animal study and promising recovery of SCI in clinical study. Therefore, in this study, we investigated how acute astrogliosis is associated with MSC transplantation in SCI rats.

\section{Materials and Methods}

\section{Surgical procedures of spinal cord injury}

All animal experiments were approved and followed the regulations of the Institutional Animal Care and Use Committee (IACUC) of Kangwon National University (IACUC NO. KW-180518-2). For the comparison of be- havioral and pathological outcome, female SD rats were anesthetized and exposed to severe SCI using the clipping compression technique $(13,14)$. After shaving and disinfected with povidone and $70 \%$ alcohol, midline incision was performed from T8 to T12 area. With serial dissection of fascia and muscle, T10 and T11 lamina were totally removed. The spinal cord injury was performed by compression of aneurysmal clips (occlusion pressure: $75 \mathrm{gm}$, Asculap, Oackville, ON, Canada). The clip was applied with full thickness of spinal cord, was left for 1 minute and then removed. For injection of NP-MSC or control, T10 and T12 vertebral bodies were fixed horizontally with forceps of fixation device.

\section{Preparation and transplantation of MSC at acute SCI of rats}

MSC were harvested from the tibia and fibula of two-week-old male Sprague-Dawley rat (Japan SLC, Inc. Hamamatsu, Japan). The MSC prepared on gelatin coated plate after removing RBCs as described previously (15). At passage 5 of MSCs, MSCs were cultured in neuronal induction media composed of DMEM $+10 \% \mathrm{FBS}, 10 \mathrm{ug} / \mathrm{L}$ basic fibroblast growth factor ( $\beta$-FGF, R\&D Systems, Inc.), $10 \mathrm{ug} / \mathrm{L}$ human epidermal growth factor (hEGF, R\&D Systems, Inc.), 1 mmol dibutyryl cyclicn AMP (dbcAMP, Sigma, St. Louis, MO) and $0.5 \mathrm{mmol}$ isobutylmethylxanthine (IBMX, Sigma, St. Louis, MO) for 7 days following by Jiang's method (16). For MSC trafficking, MSCs were tracked by CFDA tracker and then resuspended at a concentration of 10,000 cells/ul. MSC with CFDA tracker (20 ul, 200,000 cells/20 ul) were transplanted at $5 \mathrm{~mm}$ above epicenter of spinal cord after 1 hour from injury through the automatic stereotactic microinfusor (Model KDS-310; Muromachi Kikai Co., Tokyo, Japan) with $5 \mathrm{ul} / \mathrm{min}$ over 4 minutes. The needle was left in place for 5 minutes after injection.

\section{Basso Beattie and Bresnahan (BBB) locomotor scale}

Functional outcome was analyzed serially with the Basso Beattie and Bresnahan scale (BBB) at postoperative one day and weekly for four weeks. Two independent blinded examiners observed and assessed BBB for 4 min. For the comparison of behavioral outcome, twenty-five female SD rats were anesthetized and exposed to severe SCI using the clipping compression technique. MSC infused at $5 \mathrm{~mm}$ above injured spinal cord in each eight rats. Culture media or stem cell derivatives were infused at eight spinal cord injured rats for control. Nine rats were performed SCI only. 


\section{RNA isolation, real-time reverse transcription} polymerase chain reaction (RT-PCR)

According to the manufacturer's instructions, the RNeasy Mini Kit (Qiagen, Hilden, Germany) was used for extracting total mRNA from the cells and cDNA was synthesized using the ReverTra Ace qPCR RT Master Mix with gDNA remover kit (Toyobo, Osaka, Japan). Then, the quantification of the specific gene expression of Matrix metalloproteinase (MMP)-1, 2, 3, 7, 8, 9 was confirmed using a THUNDERBIRD SYBR qPCR Mix (Toyobo) under the 7500 Real-time PCR system (Applied Biosystems), and melting curve date was analyzed for identifying PCR specificity. The mRNA level was presented as $2^{-\Delta \mathrm{Ct}}$, where $\mathrm{Ct}=$ threshold cycle for target amplification, $\Delta \mathrm{Ct}=\mathrm{Ct}$ target gene (specific genes for each sample) $-\mathrm{Ct}$ internal reference $(G A P D H$ for each sample). Design of primer sequences by Primer3 software (Whitehead Institute/MIT Center for Genome Research) was conducted with information of cDNA sequences obtained from GenBank for rat and Table 1 shows information and sequences of primers.

\section{Enzyme linked immunosorbent assay (ELISA)}

Interleukin (IL)-1 $\alpha$, IL-10, C-X-C motif chemokine (CXCL)-2, CXCL-10, Tumor growth factor (TGF)- $\beta$, Tumor necrosis factor(TNF)- $\alpha$, signal transducer and activator of transcription 3 (STAT3) and nuclear factor (NF)- $\kappa$ B p65 were analyzed for evaluation of acute astrogliosis at 1,3 and $7 \mathrm{~d}$ after SCI. Protein samples from rat spinal cord were prepared Protein samples from rat spinal cord were extracted and prepared with a protease inhibitor cocktail on ice. All ELISA kits (IL-1 $\alpha$, IL-10,
CXCL-2, CXCL-10, TGF- $\beta$, TNF- $\alpha$, STAT-3, NF- $\kappa$ B pathway) were purchased from FineTest (Wuhan Fine Biological Technology Co., Ltd., Wuhan, Hubei, China) and used as per manufacturer's instructions. Microplates were read on an Epoch microplate reader (BioTek Instruments, Inc., Winooski, VT, USA). Cytokine levels were then extrapolated from a standard curve.

\section{Western blot analysis}

Protein samples from spinal cord were prepared with RIPA lysis buffer with a protease inhibitor cocktail on ice, and equal amounts of isolated protein were separated on $10 \%$ SDS-polyacrylamide gels. The separated proteins were electroblotted onto $0.45-\mu \mathrm{m}$ nitrocellulose transfer membranes. The membranes were blocked with $5 \%$ skim milk in Tris-buffered saline supplemented with $0.1 \%$ Tween 20 (TBST) buffer for $1 \mathrm{~h}$ and then probed with 1 : 1000 diluted anti- NF- $\kappa \mathrm{B}$ p65 and anti-p- NF- $\kappa \mathrm{B}$ p65 (Ser536) primary antibodies for $24 \mathrm{~h}$ at $4^{\circ} \mathrm{C}$. After 3 times washes for 10min each, polyclonal anti-rabbit HRP-conjugated secondary antibody were incubated for $1 \mathrm{~h}$ at room temperature and developed with a Pierce ECL Western Blot substrate. Band densitometry was calculated using Image $\mathrm{J}$ software analysis.

\section{Immunofluorescence staining and confocal microscopy}

Histopathologic analysis was undertaken at 7 and $28 \mathrm{~d}$ following injury. At $7 \mathrm{~d}$ after SCI, the extent of acute astrogliosis was checked by Glial fibrillary acidic protein (GFAP) staining at mid sagittal section of SCI. The cell population was checked with GFAP for glial cell, Neural/

Table 1. Oligonucleotide primers and PCR cycling conditions

\begin{tabular}{|c|c|c|c|c|c|}
\hline \multirow{2}{*}{ Genes } & \multirow{2}{*}{ GenBank number } & \multicolumn{2}{|c|}{ Primer sequence } & \multirow{2}{*}{ Size (bp) } & \multirow{2}{*}{ Temp $\left({ }^{\circ} \mathrm{C}\right)$} \\
\hline & & Sense $\left(5^{\prime}>3^{\prime}\right)$ & Anti-sense $\left(5^{\prime}>3^{\prime}\right)$ & & \\
\hline GAPDH & NM_017008.4 & ggacctcatggcctacatgg & cccctcctgttgttatgggg & 179 & 60 \\
\hline MMP1 & NM_001134530 & cttccccaaatcccatccagc & ttgagctcagcttctggcatg & 160 & 60 \\
\hline MMP2 & ВС074013 & ggccgtacaatcttcactgca & agcacctttctttgggcacaa & 182 & 60 \\
\hline MMP3 & NM_133523 & tacggctgtgtgctcatccta & gcttccctgtcatcttcagcc & 197 & 60 \\
\hline MMP7 & L24374 & ttagttgggggactgcagaca & tcctcaccatccgtccagtac & 169 & 60 \\
\hline MMP8 & NM_022221 & tggaccttcagacaaccctgt & tcaactgttctcagctgggga & 159 & 60 \\
\hline MMP9 & NM_031055 & tcattcttcagtgccggaagc & ggacacatagtgggaggagct & 183 & 60 \\
\hline $1 / 1 \alpha$ & NM_017019.1 & gacaagcctgtgttgctgaa & Gaaagctgcggatgtgaagt & 123 & 55 \\
\hline $\operatorname{Tnf} \alpha$ & NM_012675.3 & tgcctcagcctcttctcatt & cccatttgggaacttctcct & 108 & 55 \\
\hline $\operatorname{Tg} \beta$ & NM_021578.2 & tgagtggctgtctttgacg & tgggactgatcccattgatt & 146 & 55 \\
\hline $1 / 10$ & NM_012854.2 & cccagaaatcaaggagcatttg & cagctgtatccagagggtcttca & 100 & 55 \\
\hline $\mathrm{CxCl} 2$ & NM_053647.1 & agtgagctgcgctgtcaatg & gctctggatgttcttgaagtcaac & 100 & 55 \\
\hline Cxcl10 & NM_139089.1 & gggatccctctcgcaagaa & ctcagcgtctgttcatggaagt & 100 & 55 \\
\hline
\end{tabular}

Temp: Temperature. 
glial antigen (NG) 2 for oligodendroglial precursor cell (OPC), ionized calcium-binding adapter molecule (IBa) 1 for microglial cell at $7 \mathrm{~d}$ of SCI. At 4 weeks after SCI, histological outcome was determined by the relative mean percentage of spared white tissue by the Cavalieri method with Image $\mathrm{J}$ software analysis (17). For it, the axial slides were selected every 100 um from epicenter to rostral and caudal direction with Hematoxylin \& Eosin and Neurofilament staining. At 7 and 28 days after SCI, the survival and differentiation of MSC were checked with confocal microscope (Olympus FLUOVIEW - FV300).

\section{Statistical analysis}

The statistical analysis was performed by SPSS. Paired t-test was used for comparison between MSC group and control. Results are shown as means \pm SD. Statistical analysis of significance was calculated by one-way ANOVA followed by Bonferroni post hoc test for multigroup comparisons (StatView 5.0; SAS institute, Cary, NC). The variation trends of BBB scores was analyzed using ANOVA followed by Turkey's post-hoc test and their score at 28 days were represented using box-and-whisker plots with statistical analysis of Bonferroni's multiple comparison test.

\section{Results}

\section{MSC graft enhanced functional and physiological improvement after $\mathrm{SCl}$}

In order to evaluate the functional outcome of MSC grafted SCI rats, Basso Beattie and Bresnahan (BBB) locomotor scale was performed postoperatively. For 2 weeks, there was no difference of behavioral outcome between treated- and control group postoperatively. However, at 28 $\mathrm{d}$ after SCI, the BBB score of the MSC treated rats after SCI was significantly higher than SCI rats. In addition, culture media of MSCs treated rats was not significant different from SCI rats in BBB scale (Fig. 1A and 1B). We next analyzed the histological changes of the GFAP positive astrogliosis by measuring in mid sagittal section of SCI. At Day 7 after SCI, extensive astrogliosis and scar formation was observed at the injury site in MSC treated SCI rats compared with SCI or MSC-CM treated SCI rats (Fig. 2B and 2C). On the contrary of increased acute astrogliosis, MSC transplanted rats exhibited widely spared white matter at Day 28 postoperatively compared with SCI or MSC-CM transplanted SCI rats $(\mathrm{p}<0.05)$ (Fig. 2A). Moreover, grafted MSCs were observed using CFDA-SE tracking under the confocal microscopy at both Day 7 and Day 28 after SCI compared to SCI alone (Supplementary Fig. S1).
MSC transplantation decreases release of proinflammatory cytokines and level of phosphorylation of NF- $\kappa$ B p65 after SCl

In order to determine whether MSCs after SCI modulate inflammatory response, protein or mRNA levels of pro/anti-inflammatory cytokines were analyzed by ELISA or RT-PCR. As shown in Fig. 3 and Supplementary Fig. S2, protein levels of IL- $1 \alpha$, TNF- $\alpha$, TGF- $\beta$, CXCL- 2 or CXCL-10, were decreased in MSC transplanted SCI rats compared with SCI or MSC-CM transplanted SCI rats. We next determined the level of NF- $\kappa \mathrm{B}$ p65 to elucidate anti-inflammatory pathway of MSC transplanted SCI rats using western blot analysis. As shown in Fig. 4A and 4B, the phosphorylation level of the inflammatory transcriptional factor NF- $\kappa$ B p65 at Ser536 of spinal cord was

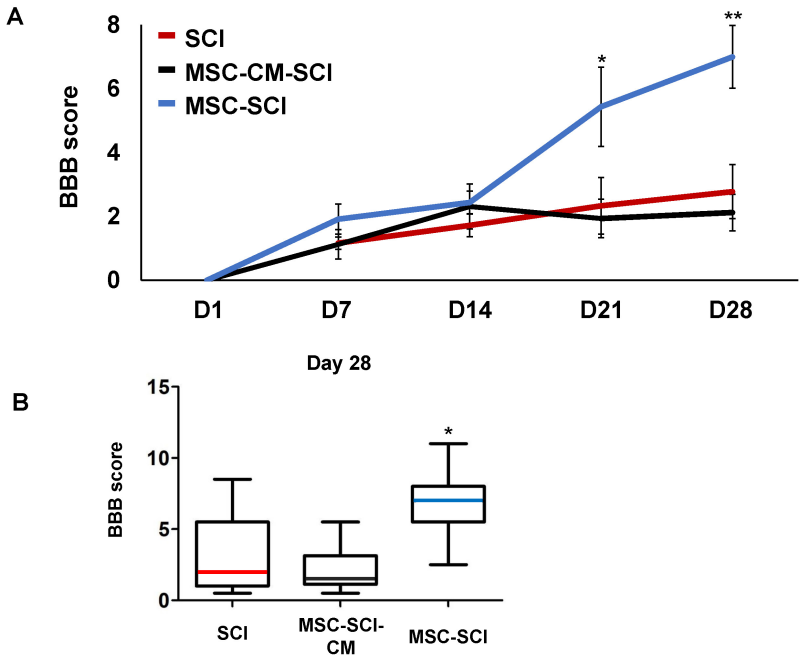

Fig. 1. Time course of behavioral recovery with acute treatment of MSC at severe $\mathrm{SCl}$. For the comparison of behavioral outcome, twenty-five female SD rats were anesthetized and exposed to severe $\mathrm{SCl}$ using the clipping compression technique. MSC were infused at eight rats and MSC culture medium (CM) at eight rats for control. Nine rats were performed $\mathrm{SCl}$. (A) Functional outcome was analyzed serially with the Basso Beattie and Bresnahan (BBB) scale at postoperative $1^{\text {st }}$ day and weekly for four weeks $\left(28^{\text {th }}\right.$ day). The statistical analysis was conducted by repeated measured analysis of variance followed by Turkey's post-hoc test. ${ }^{*} \mathrm{p}<0.05$ and ${ }^{* *} \mathrm{p}<$ 0.01 vs. score of $\mathrm{D}+1$ at each groups. From 21 days after $\mathrm{SCl}$, the rats with MSC treated group had better behavioral outcome with statistically significance than of $\mathrm{SCl}$ group. (B) The statistical analysis of box plots were accompanied by one-way analysis of variance followed by Bonferroni's multiple comparison test for comparing all pairs of columns to box-and-whisker plots of BBB score at $28^{\text {th }}$ day. Box plots display upper and lower quartiles and media values with each different color. This effects showed obvious differences between groups at $28 d(p<0.05) .{ }^{*} p<0.05$ for MSC-CM-SCl or MSC-SCl vs. SCl. D; Day. Data are presented as the mean $\pm \mathrm{SD}$. 
A

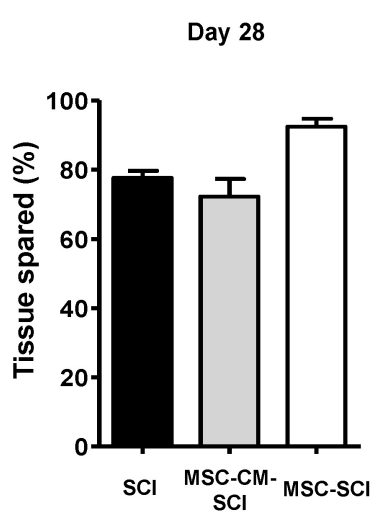

B

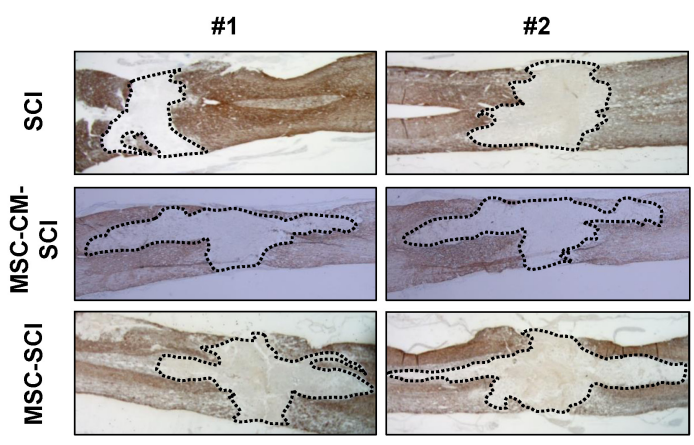

C

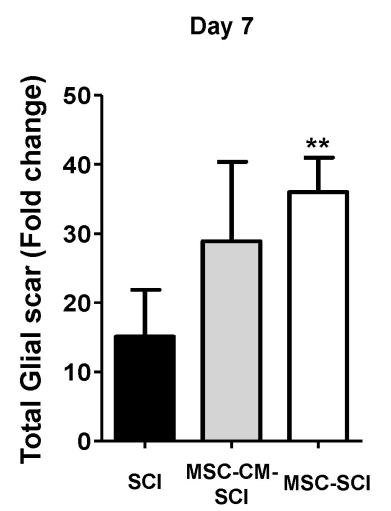

Fig. 2. Analysis of spared white mater and GFAP-positive astrogliosis with MSC treatment at acute or severe SCI. (A) At Day 28 after $\mathrm{SCl}$, histological outcome was determined by represent 2 images of each groups $(n=3)$ and their relative mean percentage of spared white tissue by the Cavalieri method. The axial slide was selected every 100 um from epicenter to rostral and caudal direction with Hematoxylin \& eosin and neurofilament staining. The mean area of spared white mater in tissue was expressed as proportional area (percentage, \%). MSC treated SCl rat had more spared white matter at the lesion epicenter compared with $\mathrm{SCl}$ or MSC-CM treated $\mathrm{SCl}$ rats. ${ }^{*} \mathrm{p}<0.05$ compared with $\mathrm{SCl}$ group. (B) The astrogliosis was described using represent 2 images of each groups $(\mathrm{n}=3$ for $\mathrm{SCl}$ or $\mathrm{MSC}-\mathrm{SCl}$ rats and $\mathrm{n}=2$ for MSC-CM-SCl rats). The extent of astrogliosis was measured by bright-filed microscopy with Glial fibrillary acidic protein (GFAP)-positive staining in mid sagittal section of $\mathrm{SCl}$ at $7 \mathrm{~d}$ after $\mathrm{SCl}$ at $\times 600$ magnification. (C) The dotted line for area occupied by GFAP- $(+)$ scar in (B) was shown as stereological quantification by image J. Data are presented as the mean $\pm \mathrm{SD}$. The area of acute astrogliosis was increased by MSC treatment in $\mathrm{SCl}$ at $7 \mathrm{~d}$ compared with $\mathrm{SCl}$ or MSC-CM treatment in $\mathrm{SCl}$. ${ }^{*} \mathrm{p}<0.01$ compared with $\mathrm{SCl}$ at each group. ${ }^{\# \#} \mathrm{p}<0.001$ compared with at $7 \mathrm{~d}$ in MSC-SCl group.

A

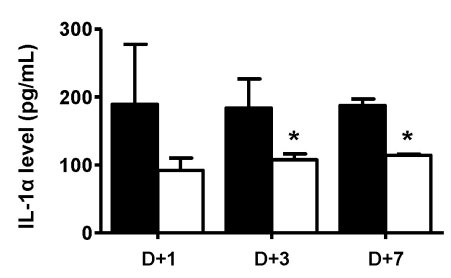

D

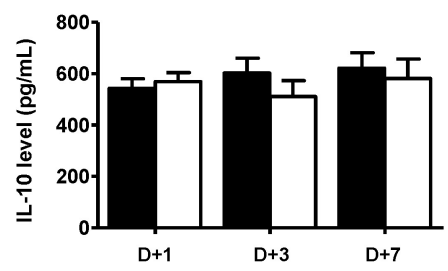

B

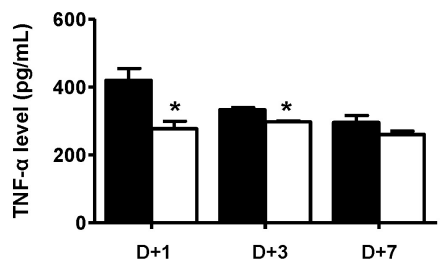

E

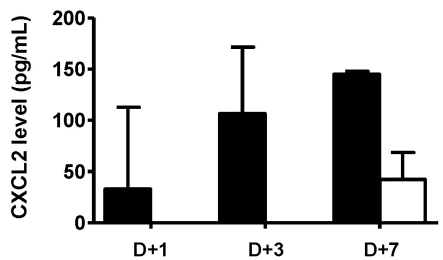

C

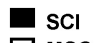

$\square$ MSC-SCI

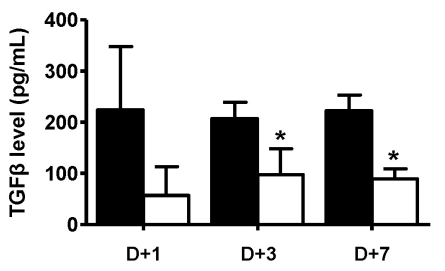

$\mathbf{F}$

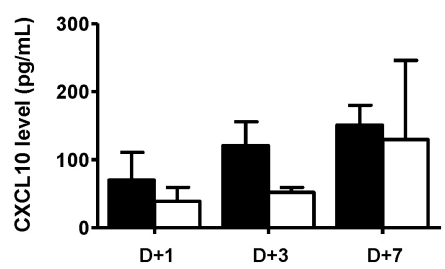

Fig. 3. The effects of MSC transplantation on cytokines/chemokine related to astrogliosis in acute $\mathrm{SCl}$. After SCl induction with or without MSC transplantation, the spine homogenates were isolated at day of first $(D+1)$, third $(D+3)$ and seventh $(D+7)$ after $S C l(n=3)$. The expression of cytokine/chemokine including (A) IL-1 $\alpha$, (B) TNF- $\alpha$, (C) TGF- $\beta$, (D) IL-10, (E) CXCL2 and (F) CXCL10 was evaluated for acute astrogliosis using ELISA analysis. Each value was calculated along with regression analysis of corresponding standard curve. MSC treatment decreased the level of cytokine/chemokine in $\mathrm{SCl}$ rat. ${ }^{*} \mathrm{p}<0.05$ for $\mathrm{MSC}-\mathrm{SCl}$ compared with $\mathrm{SCl}$ at indicated time point.

slightly decreased at 1-, 3- and $7 \mathrm{~d}$ after injury. The transcriptional level of NF- $\kappa \mathrm{B}$ p65 was confirmed in spinal cord tissue of rats by NF- $\kappa$ B p65 ELISA (Fig. 4C).

\section{Increased STAT3 and MMP2 levels in MSC treated rats} after $\mathrm{SCl}$

In astrogliosis, it is generally accepted that MMPs play 
A

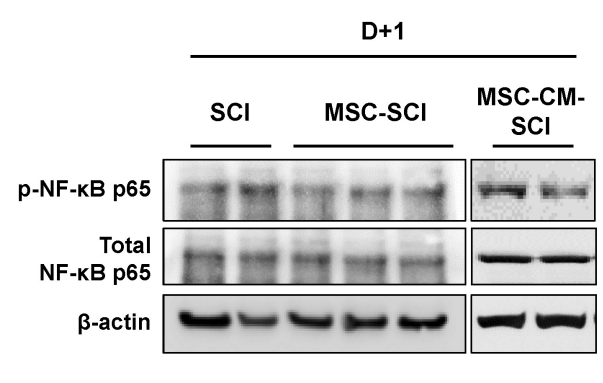

B

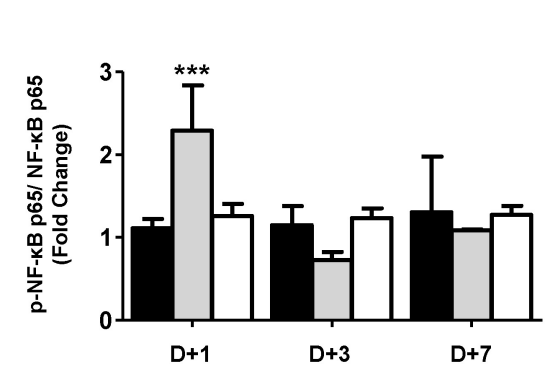

$\mathrm{D}+3$

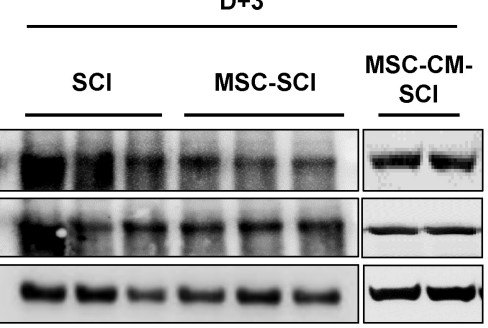

C

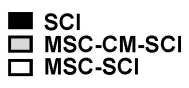

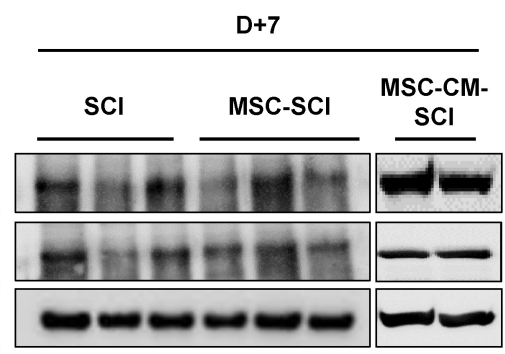

- $\mathrm{SCl}$

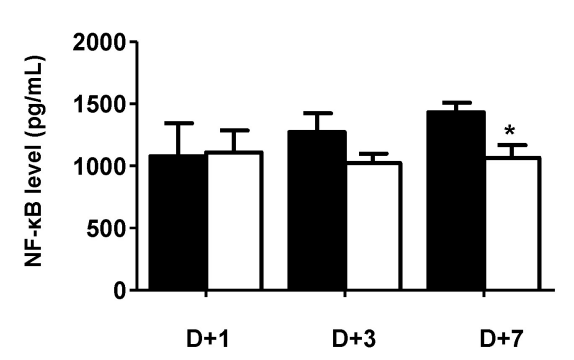

Fig. 4. The effects of MSCs transplantation on NF- $\kappa$ B pathway in acute SCl. After SCl induction with MSC or MSC-CM transplantation, the spine homogenates were isolated at 1 to $7 \mathrm{~d}$ for western blot analysis and ELISA assay to detect neuron-inflammatory pathway through activation of NF- $\kappa$ B p65 (p-NF- $\kappa$ B, 65-kDa). (A) Western blot analysis determined the phosphorylation of NF- $\kappa$ B p65 at Serine 536 residue. Total NF- $\kappa \mathrm{B}$ and $\beta$-actin were used for loading control. (B) The representative bands showed the ratio of $\mathrm{p}-\mathrm{NF}-\kappa \mathrm{B}$ p65 to total NF$\kappa \mathrm{B}$. (C) ELISA confirmed the activation of NF- $\kappa \mathrm{B}$ according to manufacturer's instruction. The value was calculated along with regression analysis of standard curve. MSC treatment decreased the activation of NF- $\kappa$ B in SCl rat. Data are presented as the mean \pm SD. ${ }^{*} p<0.05$ for MSC-CM or MSC-SCl compared with SCl at indicated time point.

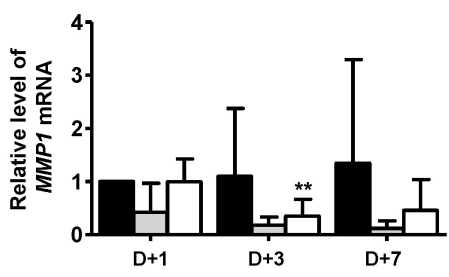

D

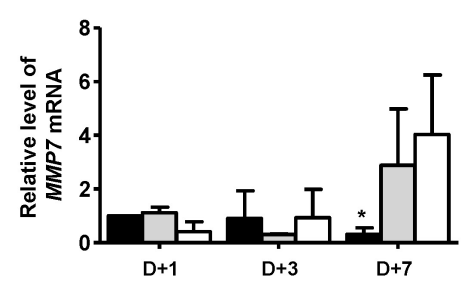

B

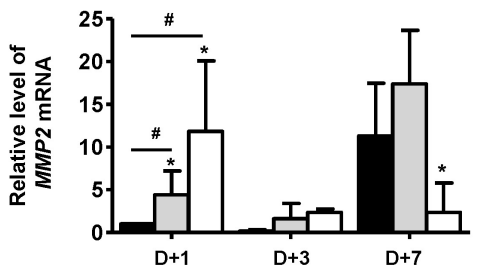

E

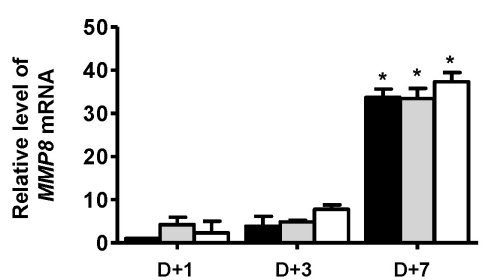

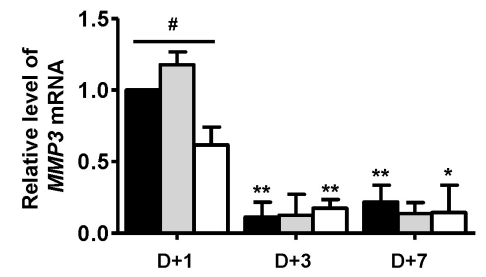

$\mathbf{F}$

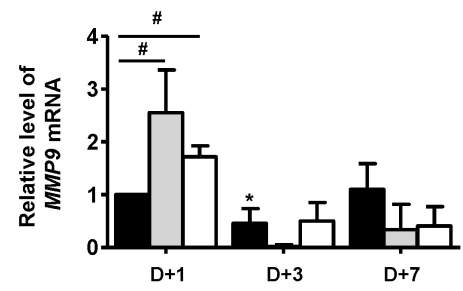

Fig. 5. The effects of MSCs transplantation on matrix metalloproteinase (MMP) and STAT3 pathway in acute SCl. After SCl induction with MSC-CM or MSC transplantation, the spine homogenates were isolated at 1 to $7 \mathrm{~d}$ in the SCl after MSC transplantation for real-time qRT-PCR. The mRNA level of (A) MMP1, (B) MMP2, (C) MMP3, (D) MMP7, (E) MMP8 and (F) MMP9 was detected in indicated time point after $\mathrm{SCl}$. The relative expression was calculated with $2^{-\triangle \triangle \mathrm{CT}}$ method and normalized with GADPH. Only MMP2 was increased in MSC-treated $\mathrm{SCl}$ rat with statically significance at $1 \mathrm{~d}$ after $\mathrm{SCl}(\mathrm{D}+1)$. Data are presented as the mean $\pm \mathrm{SD}$ of three animals for $\mathrm{MSC}-\mathrm{SCl}$ or $\mathrm{SCl}$, and two animals for MSC-CM-SCl. ${ }^{*} \mathrm{p}<0.05$ and ${ }^{* *} \mathrm{p}<0.01$ compared with $\mathrm{D}+1$ in each groups. ${ }^{*} \mathrm{p}<0.05$ for MSC-CM-SCl or MSC-SCl compared with $\mathrm{SCl}$ at indicated time point. 


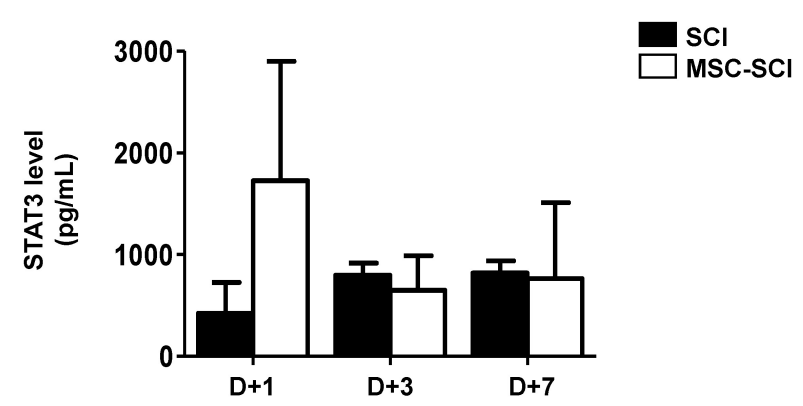

Fig. 6. The effects of MSCs transplantation on STAT3 activation in acute $\mathrm{SCl}$. After $\mathrm{SCl}$ induction with MSC-CM or MSC transplantation, the spine homogenates were isolated at 1 to $7 \mathrm{~d}$ in the $\mathrm{SCl}$ after MSC transplantation for ELISA analysis of activation of STAT3. The value was calculated along with regression analysis of standard curve. MSC treatment induces MMP2 expression and STAT3 activation in SCI rat. Data are presented as the mean \pm SD of three animals.

a key role to mobilize astrocytes and inflammatory cells by disrupting blood brain barrier (BBB) (5). Therefore, we next determined mRNA levels of MMPs in RT-PCR analysis. As shown in Fig. 5, MMP2 was significantly increased at $1 \mathrm{~d}$ in MSC transplanted rats after SCI, but not MSC-CM transplantation. We analyzed protein level of STAT3, which is a major microglial and neuro-inflammatory factor by western blot analysis. MSC grafted SCI rats exhibited increased level of STAT3 at day 1 whereas was not significantly different at 3- and $7 \mathrm{~d}$ compared with SCI or MSC-CM engrafted SCI rats (Fig. 6). Furthermore, levels of MMPs were analyzed to determine the endogenous levels of MSCs by RT-PCR analysis. As shown in Supple 2, MMP2 was significantly increased of MSCs suggesting that MSC exhibiting MMP2 is associated with its expression of MSC grafted SCI rats.

\section{Discussion}

In acute phase of central nervous system (CNS) injury, reactive astrogliosis immediately develops following microglial activation after injury (18). Sequentially, netruophil and $\mathrm{T}$ lymphocyte were infiltrated leading to recruiting large number of OPCs (18-20). In inflammatory responses at the site of injury, reactive astrocytes divide, migrate during the 2 to 7 day after injury, eventually fill the epicenter and make glial scar (12). These changes of shape, number and location of astrocytes was defined as astrogliosis which resulted in glial scar in according to the severity of injury CNS $(4,20,21)$. Classically inhibition of astrogliosis had been believed the key mechanism of MSC to SCI. In contrary to classical belief, there were filed up evidences of protective role of acute astrogliosis. Faulkner et al. (10), present astrocyte and acute gliosis improve regeneration of injured cord through decreasing scar formation. Wanner et al. (12) focus on protective role of reactive astrocyte at 5 day which confine inflammatory and fibrodic cell from heathy tissue through STAT3 pathway. Okada et al. (11) reported acute and subacute astrogliosis seclude the lesion area from healthy tissue by limiting disruption of the blood-spinal cord barrier, the amplification of an overwhelming inflammatory response and massive cellular degeneration.

Although it is widely recoginized MSC and MSC-based treatment are emering as a promising therapy in SCI, it is still controversial to make a decision an optimal timing of treatment. Regarding cellular survial, acute SCI provides a hostile environment on transplanted stem cells. Most studies have reported that the timing of treatment with MSCs was subacute phase of SCI $(7,22,23)$. In this study, we injected MSCs at early time point whether MSCs modulate astrogliosis at acute phase of SCI. It is known that gelatinase MMP2 and MMP9 are derived from astrocytes and microglia in astrogliosis (24). MMP9 is a remarkable acute marker within 24 hours (5), whereas MMP 2 is gradually increased during healing phase after SCI (5). Sassoli et al. (24) reported that MSC transplantation increased MMP2 and MMP9 expression of myoblast by paracrine effect resulting in substantially reducing muscle atrophy. Moreover, Lozito et al. (25) reported that MSCs express MMP2 at cell surface and protein secretion. In agreement with previous observation, our findings exhibited that increased level of MMP2 from isolated MSCs of rats suggesting that upregulated MMP2 of transplanted MSCs increase level of MMP2 in injured spinal cord at day 1 after SCI (Supplementary Fig. S3).

We have seen that astrogliosis increased with MSC transplantation at $7 \mathrm{~d}$ after SCI. The area of lesion core and number of astrocyte were increased in MSC treatment group compared with SCI group. Moreover, infiltration of astrocytes and microglial cells were observed exhibiting increased astrogliosis. The rats of MSC group improved motor behavioral function with BBB score and white matter sparing in rats with SCI. Veeravalli et al. (26) reported umbilical cord derived MSCs reduce the glial scar through the stimulation of MMP2 production. Renault-Mihara et al. (27) reported that MMP2 was related to STAT pathway of astrogliosis in SCI. Okada et al. (11) reported that reactive astrocytes play a pivotal role in repair of BBB and protecting neuron and oligodendroglial cells. Moreover, Anderson et al. (9) reported that inhibition of STAT3 increased area of glial scar and disturbed recovery of SCI. 
Our findings have shown the increased protein levels of STAT3 and decreased NF- $\kappa$ B p65 in SCI rats. In astrogliosis, protective pathway of STAT3 and detrimental pathway of NF- $\kappa \mathrm{B}$ has been discussed (28). In STATs pathway, STAT3 plays a key role for modulating migratory function of reactive astrocytes to recover SCI (11, 19). In this study, MMP2 was significantly increased at $1 \mathrm{~d}$ and protein level of STAT3, not NF- $\kappa \mathrm{B}$ p65, exhibited similar tendency to MMP2. These results indicate that MSC-derived MMP2- STAT3 axis is associated transiently increased astrogliosis in SCI rats. In GFAP I $\kappa$ Ba $\mathrm{KO}$ mice, the injury was dramatically improved at day 1 after SCI (21). Brambilla et al. (21) supports our findings to inhibit recovery of SCI through inhibition of NF- $\kappa \mathrm{B}$ p65 pathway.

In clinical trials, it is still remained to address MSC transplantation in SCI including quality of control in MSC as transplant, safety problem such as developing leukemia (29). Moreover, establishment of optimized animal model of SCI should be considered the clinical situation $(30,31)$. Severity of SCI is one of critical factor to outcome of stem cell therapy. Parr et al. (8) reported that neuronal stem cell produced significant functional improvement only for $27 \mathrm{~g}$ clip compression, not for $35 \mathrm{~g}$. Clinical situation, the patients with incomplete SCI regained $75 \%$ of normal function without treatment. However, preclinical study usually used a model with less consistent moderate injury model. In addition, stem cell therapy should be advanced to acute CNS injury which is critical to clinical outcome $(32,33)$. As previous mention, stem cell was usually transplanted at day 7 after SCI in animal study which is beyond critical period of human SCI (7). In addition, our previous studies have shown the method for more homogenous neuronal differentiated MSC and increased neuronal differentiation $(15,34,35)$. We transplanted MSC at acute severe SCI model to modulate astrogliosis. Acute transplanted MSC improved functional and histological outcome through increasing acute astrogliosis. For improving survival of MSC, further combined treatment needed to transplantation of acute SCI. Taken together, our findings suggest that early increased MMP2 derived from transplanted MSCs caused acute astrogliosis of injured spinal cord eventually leading to improve neurological and histrological outcine via MMP2-STAT3 axis. These data might contribute to unerstand the effect of acute treatment of MSC in SCI and its related diseases.

\section{Acknowledgments}

This work was supported by National Research Foundation (NRF) of the Korean government (2017R1D1A1A-
02019187, NRF-2017R1A2B4006197).

\section{Potential Conflict of Interest}

The authors have no conflicting financial interest.

\section{Supplementary Materials}

Supplementary data including three figures can be found with this article online at http://pdf.medrang.co.kr/ paper/pdf/IJSC/IJSC-12-s18133.pdf.

\section{References}

1. Dewan MC, Rattani A, Gupta S, Baticulon RE, Hung YC, Punchak M, Agrawal A, Adeleye AO, Shrime MG, Rubiano AM, Rosenfeld JV, Park KB. Estimating the global incidence of traumatic brain injury. J Neurosurg 2018 [Epub ahead of print]

2. Wyndaele M, Wyndaele JJ. Incidence, prevalence and epidemiology of spinal cord injury: what learns a worldwide literature survey? Spinal Cord 2006;44:523-529

3. Rossignol S, Schwab M, Schwartz M, Fehlings MG. Spinal cord injury: time to move? J Neurosci 2007;27:11782-11792

4. Sofroniew MV. Molecular dissection of reactive astrogliosis and glial scar formation. Trends Neurosci 2009;32:638-647

5. Zhang H, Adwanikar H, Werb Z, Noble-Haeusslein LJ. Matrix metalloproteinases and neurotrauma: evolving roles in injury and reparative processes. Neuroscientist 2010;16: $156-170$

6. Zhang $\mathrm{H}$, Chang $\mathrm{M}$, Hansen $\mathrm{CN}$, Basso DM, NobleHaeusslein LJ. Role of matrix metalloproteinases and therapeutic benefits of their inhibition in spinal cord injury. Neurotherapeutics 2011;8:206-220

7. Hofstetter CP, Schwarz EJ, Hess D, Widenfalk J, El Manira A, Prockop DJ, Olson L. Marrow stromal cells form guiding strands in the injured spinal cord and promote recovery. Proc Natl Acad Sci U S A 2002;99:2199-2204

8. Parr AM, Kulbatski I, Wang XH, Keating A, Tator $\mathrm{CH}$. Fate of transplanted adult neural stem/progenitor cells and bone marrow-derived mesenchymal stromal cells in the injured adult rat spinal cord and impact on functional recovery. Surg Neurol 2008;70:600-607; discussion 607

9. Anderson MA, Burda JE, Ren Y, Ao Y, O'Shea TM, Kawaguchi R, Coppola G, Khakh BS, Deming TJ, Sofroniew MV. Astrocyte scar formation aids central nervous system axon regeneration. Nature 2016;532:195-200

10. Faulkner JR, Herrmann JE, Woo MJ, Tansey KE, Doan NB, Sofroniew MV. Reactive astrocytes protect tissue and preserve function after spinal cord injury. J Neurosci 2004; 24:2143-2155

11. Okada S, Hara M, Kobayakawa K, Matsumoto Y, Nakashima Y. Astrocyte reactivity and astrogliosis after spinal cord injury. Neurosci Res 2018;126:39-43

12. Wanner IB, Anderson MA, Song B, Levine J, Fernandez A, Gray-Thompson Z, Ao Y, Sofroniew MV. Glial scar borders 
are formed by newly proliferated, elongated astrocytes that interact to corral inflammatory and fibrotic cells via STAT3-dependent mechanisms after spinal cord injury. J Neurosci 2013;33:12870-12886

13. Poon PC, Gupta D, Shoichet MS, Tator CH. Clip compression model is useful for thoracic spinal cord injuries: histologic and functional correlates. Spine (Phila Pa 1976) 2007;32:2853-2859

14. Rivlin AS, Tator CH. Effect of duration of acute spinal cord compression in a new acute cord injury model in the rat. Surg Neurol 1978;10:38-43

15. Han NR, Yun JI, Park YH, Ahn JY, Kim C, Choi JH, Lee E, Lim JM, Lee ST. Generation of priming mesenchymal stem cells with enhanced potential to differentiate into specific cell lineages using extracellular matrix proteins. Biochem Biophys Res Commun 2013;436:413-417

16. Jiang J, Lv Z, Gu Y, Li J, Xu L, Xu W, Lu J, Xu J. Adult rat mesenchymal stem cells differentiate into neuronal-like phenotype and express a variety of neuro-regulatory molecules in vitro. Neurosci Res 2010;66:46-52

17. Rabchevsky AG, Fugaccia I, Sullivan PG, Scheff SW. Cyclosporin A treatment following spinal cord injury to the rat: behavioral effects and stereological assessment of tissue sparing. J Neurotrauma 2001;18:513-522

18. Fawcett JW, Asher RA. The glial scar and central nervous system repair. Brain Res Bull 1999;49:377-391

19. Clausen F, Lorant T, Lewén A, Hillered L. T lymphocyte trafficking: a novel target for neuroprotection in traumatic brain injury. J Neurotrauma 2007;24:1295-1307

20. McTigue DM, Sahinkaya FR. The fate of proliferating cells in the injured adult spinal cord. Stem Cell Res Ther 2011;2:7

21. Brambilla R, Bracchi-Ricard V, Hu WH, Frydel B, Bramwell A, Karmally S, Green EJ, Bethea JR. Inhibition of astroglial nuclear factor kappaB reduces inflammation and improves functional recovery after spinal cord injury. J Exp Med 2005;202:145-156

22. All AH, Bazley FA, Gupta S, Pashai N, Hu C, Pourmorteza A, Kerr C. Human embryonic stem cell-derived oligodendrocyte progenitors aid in functional recovery of sensory pathways following contusive spinal cord injury. PLoS One 2012;7:e47645

23. Cusimano $M$, Biziato $D$, Brambilla $E$, Donegà $M$, Alfaro-Cervello C, Snider S, Salani G, Pucci F, Comi G, Garcia-Verdugo JM, De Palma M, Martino G, Pluchino S. Transplanted neural stem/precursor cells instruct phagocytes and reduce secondary tissue damage in the injured spinal cord. Brain 2012;135:447-460

24. Sassoli C, Nosi D, Tani A, Chellini F, Mazzanti B, Quercioli F, Zecchi-Orlandini S, Formigli L. Defining the role of mesenchymal stromal cells on the regulation of matrix metalloproteinases in skeletal muscle cells. Exp Cell
Res 2014;323:297-313

25. Lozito TP, Jackson WM, Nesti LJ, Tuan RS. Human mesenchymal stem cells generate a distinct pericellular zone of MMP activities via binding of MMPs and secretion of high levels of TIMPs. Matrix Biol 2014;34:132-143

26. Veeravalli KK, Dasari VR, Tsung AJ, Dinh DH, Gujrati M, Fassett D, Rao JS. Human umbilical cord blood stem cells upregulate matrix metalloproteinase- 2 in rats after spinal cord injury. Neurobiol Dis 2009;36:200-212

27. Renault-Mihara F, Mukaino M, Shinozaki M, Kumamaru H, Kawase S, Baudoux M, Ishibashi T, Kawabata S, Nishiyama Y, Sugai K, Yasutake K, Okada S, Nakamura M, Okano H. Regulation of RhoA by STAT3 coordinates glial scar formation. J Cell Biol 2017;216:2533-2550

28. Colombo E, Farina C. Astrocytes: key regulators of neuroinflammation. Trends Immunol 2016;37:608-620

29. Song K, Li W, Li M. Acute promyelocytic leukemia following autologous bone marrow-derived mesenchymal stem cell transplantation for traumatic brain injury: a case report. Oncol Lett 2015;10:2905-2908

30. Cheriyan T, Ryan DJ, Weinreb JH, Cheriyan J, Paul JC, Lafage V, Kirsch T, Errico TJ. Spinal cord injury models: a review. Spinal Cord 2014;52:588-595

31. Fehlings MG, Tator $\mathrm{CH}$. The relationships among the severity of spinal cord injury, residual neurological function, axon counts, and counts of retrogradely labeled neurons after experimental spinal cord injury. Exp Neurol 1995;132: 220-228

32. Bracken MB, Shepard MJ, Collins WF, Holford TR, Young W, Baskin DS, Eisenberg HM, Flamm E, Leo-Summers L, Maroon J, Marshall LF, Perot PL, Piepmeier J, Sonntag VKH, Wagner FC, Wilberger JE, Winn HR. A randomized, controlled trial of methylprednisolone or naloxone in the treatment of acute spinal-cord injury. Results of the Second National Acute Spinal Cord Injury Study. N Engl J Med 1990;322:1405-1411

33. Fehlings MG, Vaccaro A, Wilson JR, Singh A, W Cadotte D, Harrop JS, Aarabi B, Shaffrey C, Dvorak M, Fisher C, Arnold P, Massicotte EM, Lewis S, Rampersaud R. Early versus delayed decompression for traumatic cervical spinal cord injury: results of the Surgical Timing in Acute Spinal Cord Injury Study (STASCIS). PLoS One 2012;7:e32037

34. Lee H, Han NR, Hwang JY, Yun JI, Kim C, Park KH, Lee ST. Gelatin Directly enhances neurogenic differentiation potential in bone marrow-derived mesenchymal stem cells without stimulation of neural progenitor cell proliferation. DNA Cell Biol 2016;35:530-536

35. Park YH, Yun JI, Han NR, Park HJ, Ahn JY, Kim C, Choi JH, Lee E, Lim JM, Lee ST. Mass production of early-stage bone-marrow-derived mesenchymal stem cells of rat using gelatin-coated matrix. Biomed Res Int 2013;2013:347618 